\title{
Agroinfiltration of grapevine leaves for fast transient assays of gene expression and for long-term production of stable transformed cells
}

\author{
Michela Zottini - Elisabetta Barizza $\cdot$ Alex Costa • \\ Elide Formentin - Cristina Ruberti - Francesco Carimi · \\ Fiorella Lo Schiavo
}

Received: 31 October 2007/Revised: 19 December 2007/Accepted: 18 January 2008/Published online: 7 February 2008

(c) Springer-Verlag 2008

\begin{abstract}
Agrobacterium-mediated transient assays for the analysis of gene function are used as alternatives to genetic complementation and stable plant transformation. Although such assays are routinely performed in several plant species, they have not yet been successfully applied to grapevines. We explored genetic background diversity of grapevine cultivars and performed agroinfiltration into in vitro cultured plants. By combining different genotypes and physiological conditions, we developed a protocol for efficient transient transformations of selected grapevine cultivars. Among the four cultivars analyzed, Sugraone and Aleatico exhibited high levels of transient transformation. Transient expression occurred in the majority of cells within the infiltrated tissue several days after agroinfiltration and, in a few cases, it later spread to a larger portion of the leaf. Three laboratory strains of Agrobacterium tumefaciens with different virulence levels were used for agroinfiltration assays on grapevine plants. This method promises to be a powerful tool to perform subcellular localization analyses. Grapevine leaf tissues were transformed with fluorescent markers targeted to cytoplasm
\end{abstract}

Communicated by D. Somers.

Electronic supplementary material The online version of this article (doi:10.1007/s00299-008-0510-4) contains supplementary material, which is available to authorized users.

M. Zottini $(\bowtie) \cdot$ E. Barizza $\cdot$ A. Costa $~ E$ E. Formentin .

C. Ruberti · F. Lo Schiavo

Dipartimento di Biologia, Università Degli Studi di Padova,

Via U. Bassi 58/B, 35131 Padua, Italy

e-mail: mzottini@bio.unipd.it

F. Carimi

Istituto di Genetica Vegetale, Palermo (CNR),

Corso Calatafimi 414, 90128 Palermo, Italy (free GFP and mRFP1), endoplasmatic reticulum (GFP::HDEL), chloroplast (GAPA1::YFP) and mitochondria $(\beta:: \mathrm{GFP})$. Confocal microscope analyses demonstrated that these subcellular compartments could be easily visualized in grapevine leaf cells. In addition, from leaves of the Sugraone cultivar agroinfiltrated with endoplasmic reticulum-targeted GFP-construct, stable transformed cells were obtained that show the opportunity to convert a transiently transformed leaf tissue into a stably transformed cell line.

Keywords: Vitis vinifera Plant cell cultures .

Agrobacterium - Transformation - Subcellular localization

\section{Introduction}

The grapevine (Vitis vinifera L.) is one of the most important crops in Mediterranean areas. However, its use as a model organism is limited by it being a perennial plant with a long reproductive cycle. The recent delivery of the complete genome sequence (The French-Italian Public Consortium for Grapevine Genome Characterization 2007) stimulates the search for post-genomic tools capable to accelerate the acquisition of knowledge of some of its most important agronomic characteristics. Among these, the gene ectopic expression represents an important assay for the functional characterization of DNA sequences. Agrobacterium-mediated transient assays had become the favourite choice in many functional analyses and, in particular, leaf agroinfiltration represents nowadays an easy and not invasive technique (Wroblewski et al. 2005). In the past decade, this approach has been employed in different plant species (Kapila et al. 1997) to study silencing mechanisms of resident genes (Schöb et al. 1997), to in 
vivo characterize promoter and transcription factors (Yang et al. 2000), for investigating sub-cellular localization and intracellular trafficking of gene products (Batoko et al. 2000, daSilva et al. 2004), and as a tool for rapid biophysical analysis of plant ion channels (Latz et al. 2007).

Transformation and regeneration of grapevine plants have been achieved (Mezzetti et al. 2002), demonstrating that grapevine is not recalcitrant to Agrobacterium infection, but this approach is time-consuming and takes several months to produce transgenic plants suitable for analyses. On the other hand, grapevine leaf agroinfiltration has not been reported to be successful to date (Wroblewski et al. 2005). In this paper, the authors clearly demonstrated, in several plant species, that the genetic background and plant growth conditions are key factors in performing successfully leaf agroinfiltration experiments. In addition, the compatibility of A. tumefaciens strains with the plant species represents the other important variable to be considered in this kind of assay.

The studies reported in this paper establish an efficient transient assay for grapevines. We investigated the genotype effect and plant growth conditions of four grapevine cultivars and the effect of three laboratory bacterial strains to induce transient gene expression. Particularly, we used laboratory strains of A. tumefaciens originate mainly from two wild isolates (Hellens et al. 2000b), namely GV3101 and AGL1 from the C58 strain (Hamilton and Fall 1971) and LBA4404 from the Ach5 strain (Hoekema et al. 1983). We followed leaf transformation events by monitoring the expression of green fluorescent protein (GFP) targeted to cytoplasm, endoplasmic reticulum (ER) and mitochondria, the expression of yellow fluorescent protein (YFP) targeted to chloroplasts and the expression of red fluorescent protein (mRFP1) targeted to cytoplasm.

As a result, reliable, high levels of gene expression were achieved in two grapevine cultivars and compatible strains of A. tumefaciences were identified. In addition, we show that it is possible to obtain a stable transformed cultured cell line starting from agroinfiltrated leaf tissues.

\section{Material and methods}

Plant material and growth conditions

\section{In vitro grown plants}

Somatic embryos were obtained from stigma and style tissues of Sugraone, Aleatico, Moscato Giallo and Aglianico Vitis vinifera plants as described by Carimi et al. (2005). Individual somatic embryos, about $4 \mathrm{~mm}$ in length, were collected and allowed to germinate in Petri dishes containing MS (Murashige and Skoog medium, Duchefa,
The Netherlands) (Murashige and Skoog 1962) solid medium $(0.8 \%$ plant agar, Duchefa) supplemented with $88 \mathrm{mM}$ sucrose (Fig. 1a). Plantlets were multiplied by clonal propagation and maintained (30-day intervals) in Microbox Containers (Duchefa) in half strength MS solid medium ( $0.8 \%$ plant agar, Duchefa), supplemented with $44 \mathrm{mM}$ (Fig. 1b) sucrose. Plants were incubated in a growth chamber at $25 \pm 1{ }^{\circ} \mathrm{C}$ under a 16-h day length, and a photosynthetic photon flux of $35 \mu \mathrm{mol} \mathrm{m}{ }^{-2} \mathrm{~s}^{-1}$ Osram cool-white $18 \mathrm{~W}$ fluorescent lamps.

\section{In vitro establishment of greenhouse-grown plants}

Following the protocol described by Chee and Pool (1987), nodal explants and apical shoots, $1.5 \mathrm{~cm}$ long, of Sugraone cultivar were sterilized $10 \mathrm{~min}$ with $3 \%$ hypochlorite and $0.1 \%$ Tween 20 and rinsed with sterile water. Sterilized explants were placed on C2D medium (Duchefa) supplemented with $4.4 \mu \mathrm{M}$ 6-benzylaminopurine (SIGMA) and $0.49 \mu \mathrm{M}$ indol-3-butyric acid (SIGMA). After shoot emergence, explants were transferred for rooting to half strength MS solid medium $(0.8 \%$ plant agar, Duchefa) supplemented with $44 \mathrm{mM}$ sucrose. Plants were incubated in growth chamber in the conditions previously described.

\section{Cell cultures}

Stable transformed grapevine cell lines were obtained from agroinfiltrated leaf explants, washed with $\mathrm{PPM}^{\mathrm{TM}}$ solution (Micropoli, Italy) and incubated on selective B5 (Gamborg B5 medium, Duchefa; Gamborg et al. 1968) solid medium (0.8\% plant agar, Duchefa) supplemented with $2.26 \mu \mathrm{M}$ 2,4-dichlorophenoxy-acetic acid (SIGMA), cefotaxime $200 \mu \mathrm{g} / \mathrm{ml}$, kanamycin $50 \mu \mathrm{g} / \mathrm{ml}$. Transformed callus was maintained on the same solid medium with a subculture cycle of 3 weeks. After several subculture cycles (at least six), aliquots of stable transformed callus were transferred to Erlenmeyer flasks in liquid B5 medium supplemented with 2,4-dichlorophenoxy-acetic acid $2.26 \mu \mathrm{M}$ without selection. The suspension cultures were subcultured in fresh medium every week and maintained in a climate growth chamber at $25 \pm 1^{\circ} \mathrm{C}$ on an orbital shaker (80 rpm).

\section{Genetic materials}

For the expression of free GFP and GFP targeted to the mitochondria, the cDNA coding sequences of GFP and $\beta::$ GFP were subcloned from the $\beta::$ GFP plasmid (Zhao et al. 2000; Duby et al. 2001) to the pBI121 binary vector 
(Clontech Laboratories, USA) by replacing the $\beta$-glucuronidase cDNA sequence. For sub-cloning, the free GFP and the $\beta$-GFP fusion construct into the $\mathrm{pBI} 121$ binary vector the BamHI/SacI restriction sites and BglII/SacI restriction sites were used, respectively. The pBIN-m-gfp5ER binary vector, harbouring a GFP targeted to the endoplasmic reticulum, was provided by Dr. Jim Haseloff (University of Cambridge) (Haseloff et al. 1997). For the yellow fluorescent protein (YFP) chloroplast localization, we amplified the full coding sequence of the Arabidopsis thaliana glyceraldehyde 3 phosphate dehydrogenase A1 (At3g26650, Marri et al. 2005) using specific $5^{\prime}$ and $3^{\prime}$ primers where NcoI sites were introduced. The amplicon was digested with NcoI and subcloned upstream of the YFP sequence into the pAVA554 vector provided by Prof. Albrecht von Arnim (http://www.bio.utk.edu/vonarnim/ vectors.html) (von Arnim et al. 1998). In order to obtain a binary vector for the expression of free monomeric red fluorescent protein mRFP1 (Campbell et al. 2002) in plant cells, the mRFP1 cDNA was amplified by PCR from the pRSET-mRFP1 vector provided by Prof. Roger Tsien (UCSD, La Jolla) (Campbell et al. 2002) using specific $5^{\prime}$ and $3^{\prime}$ primers where the NcoI/BglII sites were introduced. The mRFP1 amplicon was then digested with BglII and a partial digestion was performed with NcoI and the band corresponding to the mRFP1 size was subcloned in the pAVA554 vector by replacing the yellow fluorescent protein (YFP) coding sequence. The pAVA554 vectors were therefore composed of the double $35 \mathrm{~S}$ promoter, the translational enhancer sequence of TEV, the GAPA1::YFP or mRFP1 coding sequences and the $35 \mathrm{~S}$ terminator. The vectors were sequenced to verify that no mistakes were introduced by PCR amplification. The entire expression cassettes of both GAPA1::YFP and mRFP1 were thereafter isolated from the pAVA554 modified vectors by digestion with $\mathrm{KpnI} / \mathrm{SacI}$, and $\mathrm{KpnI} / \mathrm{SmaI}$, respectively, and subcloned in the pGreen 0029 binary vector (Hellens et al. 2000a).

\section{Agrobacterium tumefaciens strains}

Competent cells of A. tumefaciens LBA4404, GV3101 and AGL1 strains were prepared according to Main et al. (1995) and the binary vectors were introduced by electroporation. For the use of pGreen-derived vectors we cotransformed the A. tumefaciens cells with the pSoup vector (Hellens et al. 2000a). The growth of bacteria was optimized by growing in YEP medium (Bacto-Trypton, $10 \mathrm{~g} / \mathrm{l}$; yeast extract, $10 \mathrm{~g} / \mathrm{l}, \mathrm{NaCl}, 5 \mathrm{~g} / \mathrm{l} ; \mathrm{pH}$ 7.0) the GV3101 and AGL1 strians or in YEB medium (beef extract, $5 \mathrm{~g} / \mathrm{l}$; yeast extract, $1 \mathrm{~g} / \mathrm{l}$; Bacto-Peptone $5 \mathrm{~g} / \mathrm{l}$; sucrose $5 \mathrm{~g} / \mathrm{l}$; $\mathrm{MgSO}_{4} \cdot 7 \mathrm{H}_{2} \mathrm{O} 5 \mathrm{mM}$; pH 7.2) for the LBA4404 strain. The media were supplemented with specific antibiotics (rifampicin $100 \mu \mathrm{g} / \mathrm{ml}$, streptomycin $50 \mu \mathrm{g} / \mathrm{ml}$, kanamycin $50 \mu \mathrm{g} / \mathrm{ml}$ for LBA4404; rifampicin $100 \mu \mathrm{g} / \mathrm{ml}$, gentamycin $50 \mu \mathrm{g} / \mathrm{ml}$, kanamycin $50 \mu \mathrm{g} / \mathrm{ml}$ for $\mathrm{GV} 3101$; rifampicin $100 \mu \mathrm{g} / \mathrm{ml}$, kanamycin $50 \mu \mathrm{g} / \mathrm{ml}$ for AGL1). Tetracyclin $(5 \mu \mathrm{g} / \mathrm{ml})$ was added in the cultures media when A. tumefaciens harbouring pGreen vectors were used.

\section{Grapevine leaf agroinfiltration}

Two days prior to agroinfiltration, single colonies of $A$. tumefaciens growing on agar plates were inoculated in $5 \mathrm{ml}$ of YEP or YEB liquid medium supplemented with specific antibiotics. The bacterial cultures were incubated for 2 days at $28-30^{\circ} \mathrm{C}$ at $200 \mathrm{rpm}$ on an orbital shaker. New bacteria cultures were started two days later by inoculating fresh medium with the old suspension cultures $(1 / 200$ ratio, v/v). The new cultures were grown under the same conditions for an additional day. After $24 \mathrm{~h}, 2 \mathrm{ml}$ of each bacteria cultures were transferred to Eppendorf tubes and pelletted by centrifugation at $1,500 \mathrm{~g}$ for $4 \mathrm{~min}$ in a micro-centrifuge at room temperature. The pellets were washed twice with $2 \mathrm{ml}$ of infiltration buffer [50 mM MES pH 5.6, $2 \mathrm{mM} \mathrm{Na}_{3} \mathrm{PO}_{4}$, $0.5 \%$ glucose $(\mathrm{w} / \mathrm{v})$ and $100 \mu \mathrm{M}$ acetosyringone (Aldrich, Italy)]. The bacteria suspensions were diluted with infiltration buffer to a final $\mathrm{OD}_{600}$ of 0.2 and allowed to grow for $1-2 \mathrm{~h}$ at $25^{\circ} \mathrm{C}$ in the dark, with continuous gentle shaking before using them for agroinfiltration experiments. The inocula were delivered to the lamina tissues of grapevine leaves by gentle pressure infiltration through the stomata of the lower epidermis by using a 1-ml syringe without a needle. Young leaves prior to full expansion (about $2 / 3$ of the full size) were used. For experiments requiring co-infection of more than one construct, bacteria strains containing the constructs were mixed before performing the leaf infection, with the inoculum of each construct adjusted to a final $\mathrm{OD}_{600}$ of 0.2. After infiltration the plants were transferred in a growth chamber under standard growth conditions.

Evaluation of gene expression trough fluorescent proteins

GFP-dependent fluorescence was analyzed at 6 and 12 days after infection. Routinely, the expression pattern and intracellular distribution of GFP fluorescence were assessed using an epifluorescence stereo microscope before confocal microscopy. Pieces of leaf were randomly cut from the infected areas and mounted on slides for microscopic observations. Confocal microscope analyses were 
performed using a Nikon PCM2000 (Bio-Rad, Germany) laser scanning confocal imaging system. For GFP and YFP detection, excitation was at $488 \mathrm{~nm}$ and emission between $515 / 530$ and 525/540 nm, respectively. For the chlorophyll detection, excitation was at $488 \mathrm{~nm}$ and detection over $570 \mathrm{~nm}$. For mRFP1 detection, excitation was set at $548 \mathrm{~nm}$ and emission $573 \mathrm{~nm}$. Image analysis was done with the ImageJ bundle software (http://rsb.info.nih.gov/ij/).

\section{Results and discussion}

Analysis of transformation events

Grapevine leaves were agroinfiltrated in sterile conditions (Fig. 1c, d) and after 6 and 12 days were cut from the plant and analysed under a stereo microscope equipped with white and UV light.

Figure 2 (upper panel) shows the results obtained by agroinfiltration of Sugraone leaves. As can be seen by images taken under UV light, all Agrobacterium strains were able to induce transformation as shown by bright green spots detected in the infiltrated areas. The transformation events were detected 6 days after infiltration and were still visible after 12 days. By comparing the fluorescent intensities of the regions expressing the GFP, an increase of the fluorescent signal from 6 to 12 days was observed. The three Agrobacterium strains gave very similar results. Interestingly, agroinfiltration of Sugraone leaves led to fluorescent signals only inside the syringe contact zones and not in the surrounding areas (Fig. 2, upper panel). The bright field images show that mechanical damage due to the pressure applied during the manipulation led in some cases to tissue browning or even to restricted areas of necrosis. Nevertheless, a large number of transformed cells were still detected in these areas.

The same agroinfiltration experiments were performed on the Aleatico cultivar, which also showed positive results with all three Agrobacterium strains. The lower panel of Fig. 2 shows the presence of large bright fluorescent areas that do not correspond to the syringe contact zone. This might be due to a different leaf texture of Aleatico versus
Sugraone leaves and/or a different biological response to Agrobacterium (Melotto et al. 2006).

As in the case of Sugraone leaves, leaf tissue was sometimes damaged during agroinfiltration, but no browning areas in the transformed regions outside the contact zones were observed. The fluorescence intensity of the transformed areas increased from 6 to 12 days. All three Agrobacterium strains gave similar results.

We thereafter agroinfiltrated leaves of Moscato Giallo and Aglianico regenerated plants under the same experimental conditions used previously. Leaves of Moscato Giallo infiltrated with the GV3101 Agrobacterium strain occasionally gave bright spots due to GFP fluorescence such as those reported in Fig. 3 (upper panel). Similar results were obtained with the other two bacteria strains (data not shown). With the Aglianico cultivar transformation events were never observed. However, background fluorescence (Fig. 3 lower panel) due to cell wall thickening was detectable, probably due to a specific response to the Agrobacterium. The browning and the necrotic areas detected on the leaves of these two cultivars were comparable with what observed on Sugraone and Aleatico leaves after inoculation.

The fact that different cultivars showed different susceptibility towards Agrobacterium infection clearly implies an effect of the plant's genetic background. It has been previously reported that some species are recalcitrant to Agrobacterium transformation by agroinfiltration (Citovsky et al. 2007 and reference herein), and in Arabidopsis it has been shown that a specific LRR receptor kinase is involved in this interaction (Zipfel et al. 2006). In particular, it was reported that agroinfiltration did not occur in grapevine plants grown in a greenhouse, where instead a clear necrotic response was induced (Wroblewski et al. 2005). In our experimental system, the use of in vitro cultured plants seems to help overcome necrotic responses (apart from the mechanically-induced ones), since we were able to obtain reliable leaf transformation events using Agrobacterium strains with different virulence levels (Hellens et al. 2000b; Jones et al. 2005). However, the in vitro culture conditions were not the only determining factor in this process. The genetic background was of
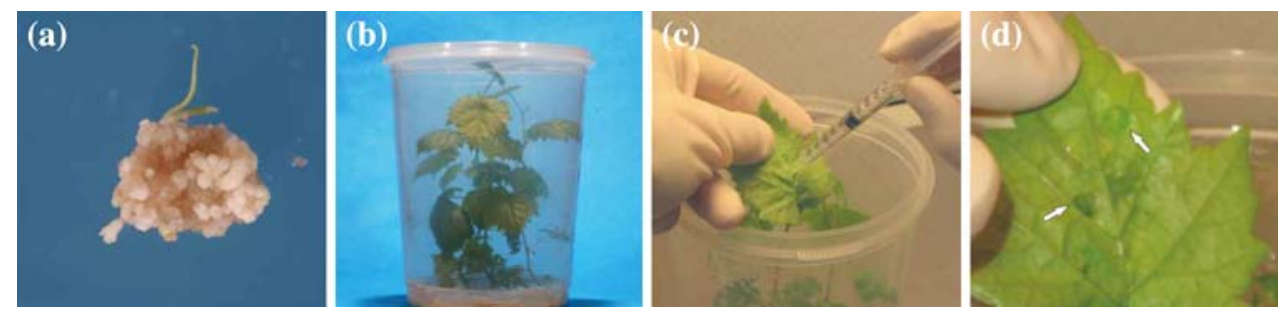

Fig. 1 Agroinfiltration performed on in vitro grapevine plants. a Embryogenic callus; $\mathbf{b}$ in vitro regenerated plants; $\mathbf{c}$ agroinfiltration performed on the leaf lower surface using 1-ml syringe with no needle; $\mathbf{d}$ multiple infiltrations conducted on a single leaf (arrows) 
Fig. 2 Analysis of Sugraone (upper panel) and Aleatico (lower panel) leaves agroinfiltrated with $A$. tumefaciens harbouring cytosolic GFP construct. Leaves were observed under a stereo microscope equipped with white and UV light, 6 and 12 days after infiltration with three different A. tumefaciens strains. First and third columns show bright field images of agroinfiltrated leaves. Second and forth columns show the same leaf regions acquired under UV light where specific GFP fluorescence can be detected as bright green spots. Each picture is representative of at least 15 agroinfiltrations

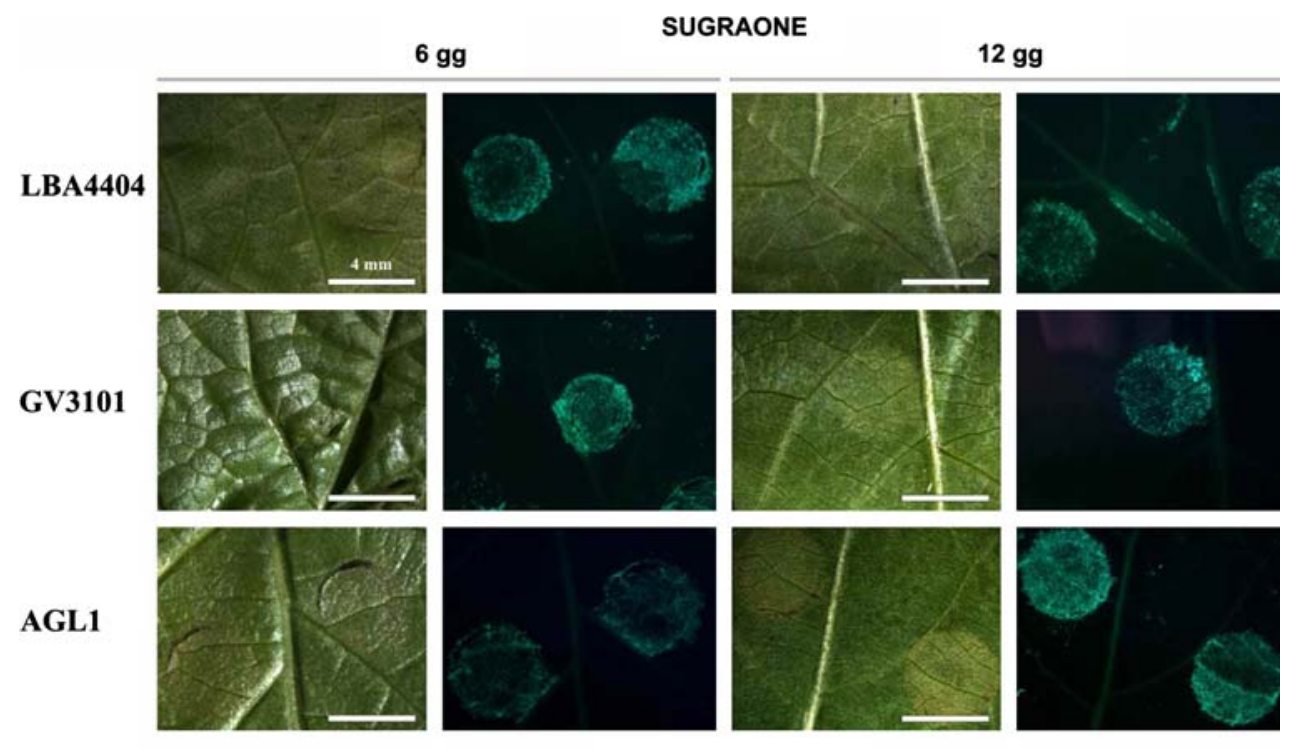

ALEATICO

LBA4404
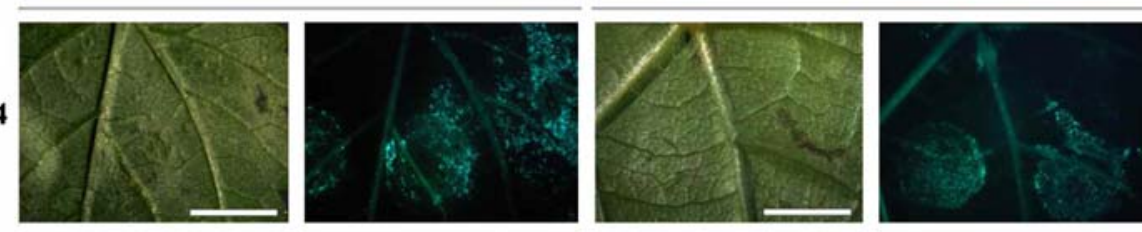

GV3101
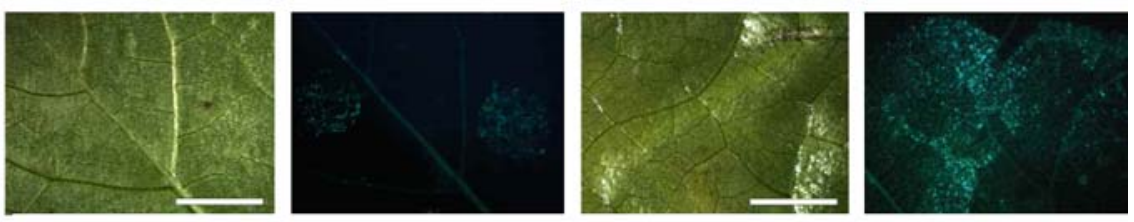

AGL1

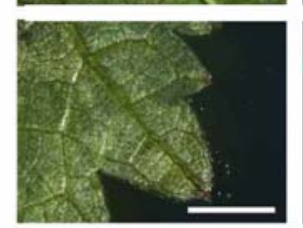

Bright field

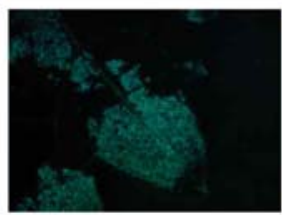

Fluorescence

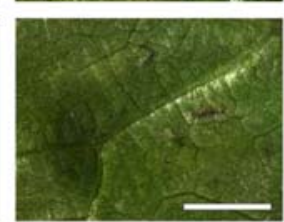

Bright field

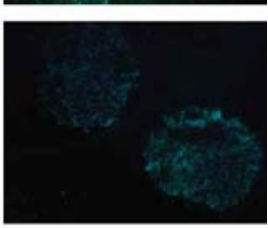

Fluorescence
Fig. 3 Analysis of Moscato Giallo (upper panel) and Aglianico (lower panel) leaves agroinfiltrated with $A$. tumefaciens harbouring the cytosolic GFP construct. Leaves were observed under a stereo microscope equipped with white and UV light, 6 and 12 days after infiltration with GV3101 A. tumefaciens strain. First and third columns show bright field images of agroinfiltrated leaves. Second and fourth columns show the same leaf regions acquired under UV light. Each picture is representative of at least 15 agroinfiltrations

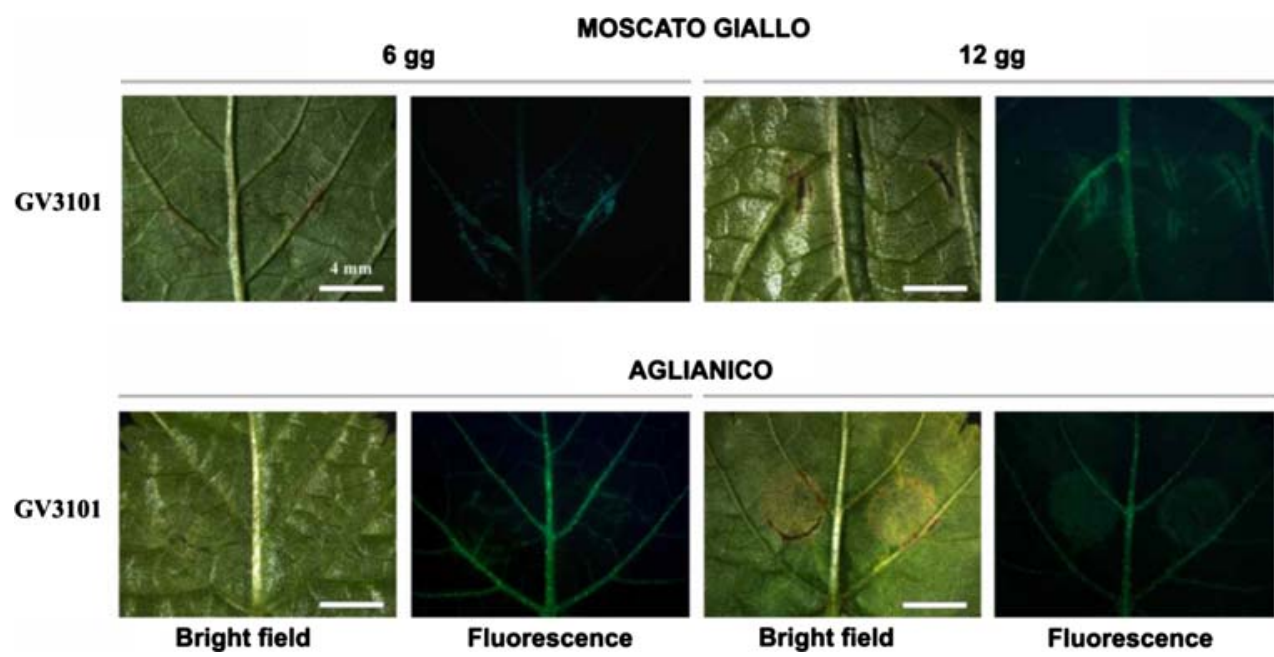


fundamental importance for successful grapevine agroinfiltration, as demonstrated by the fact that even if the Aglianico cultivar was obtained from in vitro regenerated plants, it was recalcitrant to agroinfiltration.

In order to evaluate the effect of in vitro growth conditions on this transformation protocol, we performed agroinfiltration experiments on Sugraone plants grown in greenhouse. These plants were not susceptible to transformation (Fig. 4 upper panel) even if various bacterial suspension density were used, or the plants were kept under high humidity conditions to mimic in vitro growth. Nodal explants of these greenhouse grown Sugraone plants (see "Material and methods") were transferred to in vitro culture, for a time sufficient to develop new leaves. When the plants reached the appropriate growth stage, agroinfiltration assay was performed successfully (Fig. 4 lower panel), thus confirming the important role played by the in vitro culture growth conditions.

\section{Subcellular localization of transient expression}

In order to validate our agroinfiltration protocol for successful subcellular localization analyses, we agroinfiltrated grapevine leaves with Agrobacterium harbouring GFP constructs targeted to different subcellular compartments. Due to the high reproducibility of transformation obtained with the Sugraone cultivar and the GV3101 strain, we present results obtained with this combination. We used (1) free GFP localized in the cytoplasm that is also able to diffuse to the nucleus (Brandizzi et al. 2002); (2) a GFP::HDEL construct to visualize the endoplasmic reticulum (ER) since the HDEL signal was shown to efficiently

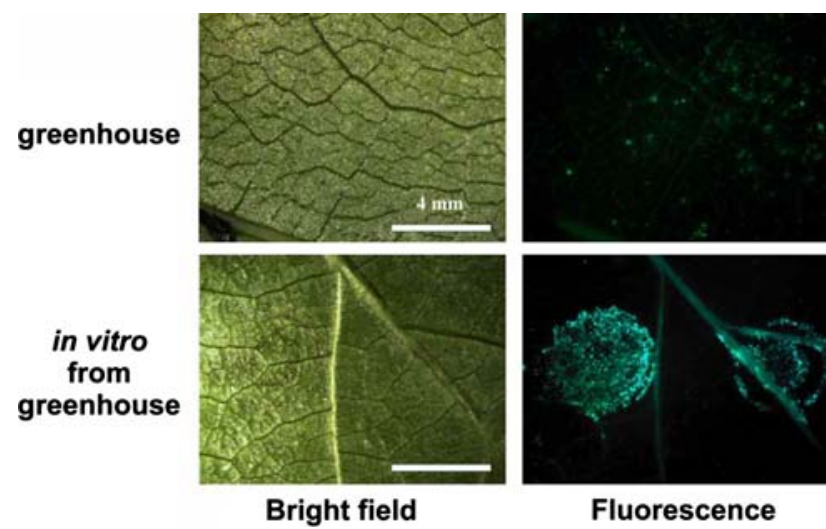

Fig. 4 Effect of growth conditions on transformation attitude. Leaves from Sugraone plants grown in different conditions (greenhouse, in vitro from greenhouse) were agroinfiltrated with GV3101 A. tumefaciens strain harbouring cytosolic GFP construct. The leaves were observed under a stereo microscope equipped with white and UV light, 6 days after infiltration. Each picture is representative of at least 15 agroinfiltrations retain proteins in the ER lumen of plant cells (Haseloff et al. 1997; Brandizzi et al. 2002); (3) GFP fused to the presequence of the mitochondrial $\beta$-subunit of the F1-ATP synthase isolated from Nicotiana plumbaginifolia to localize mitochondria (Duby et al. 2001); (4) YFP fused to chloroplastic glyceraldehyde-3-phosphate dehydrogenase A1 (GAPA1) isolated from Arabidopsis thaliana (At3g26650) to target chloroplasts (Marri et al. 2005).

In Fig. 5, leaves of Sugraone cultivar are shown 6 days after agroinfiltration with GV3101 Agrobacterium harbouring the above-listed vectors. As expected, epidermal cells expressing free GFP (Fig. 5a, b) showed a fluorescence diffuse signal in the cytoplasm and nucleoplasm. The presence of the signal in the cytoplasm was also confirmed by detection of clear cytoplasmic streaming (data not shown).

In leaves expressing the GFP::HDEL fusion protein, a signal localized to cell endomembranes was detected (Fig. 5c, d). In this latter case, the fluorescence intensity was lower than that observed with the free GFP. Typical ER polygonal structures present in the plant cells could be easily observed, as well as staining of the nuclear envelope (NE) (arrow in Fig. 5d), as previously reported (Haseloff et al. 1997; Brandizzi et al. 2002).

Figure $5 \mathrm{e}, \mathrm{f}$ shows the cells transformed with the $\beta:$ GFP fusion protein. In this case, as expected, highly mobile punctuate structures throughout in cytoplasm were detected. No fluorescence was observed in either the cytoplasm or nucleoplasm. This result confirms the specific GFP import into mitochondria, and the clear and easy detection of these organelles in leaf grapevine cells.

When the leaves were infiltrated with the Agrobacterium GV3101 harbouring the GAPA1::YFP, a clear fluorescence signal into the chloroplasts was detected as shown in Fig. $5 \mathrm{~g}$, h by an overlapping signal of YFP and chlorophyll. This is relevant because it demonstrates unequivocally that not only epidermal cells are successfully transformed by agroinfiltration, but also that mesophyll cells which, at variance with epidermal cells, are rich in chloroplasts (Taiz and Zeiger 2006).

The availability of different fluorescent proteins, with characteristic excitation and emission spectra, allow the simultaneous visualization of two or more different fluorophores in the same transformed cell. Taking advantage of this technology, we co-agroinfiltrated grapevine leaves with a mixture of two GV3101 Agrobacterium populations, harbouring the $\beta:$ GFP construct or the free mRFP1 fluorescent protein (Campbell et al. 2002). Figure 5i, 1 shows typical results obtained in co-agroinfiltrated Sugraone leaf cells. It can be observed that co-expression does not occur in all cells (Fig. 5i), but was easily detected in many (Fig. 51). In this latter figure, the presence of green punctuate structures, that represent mitochondria, and a red 


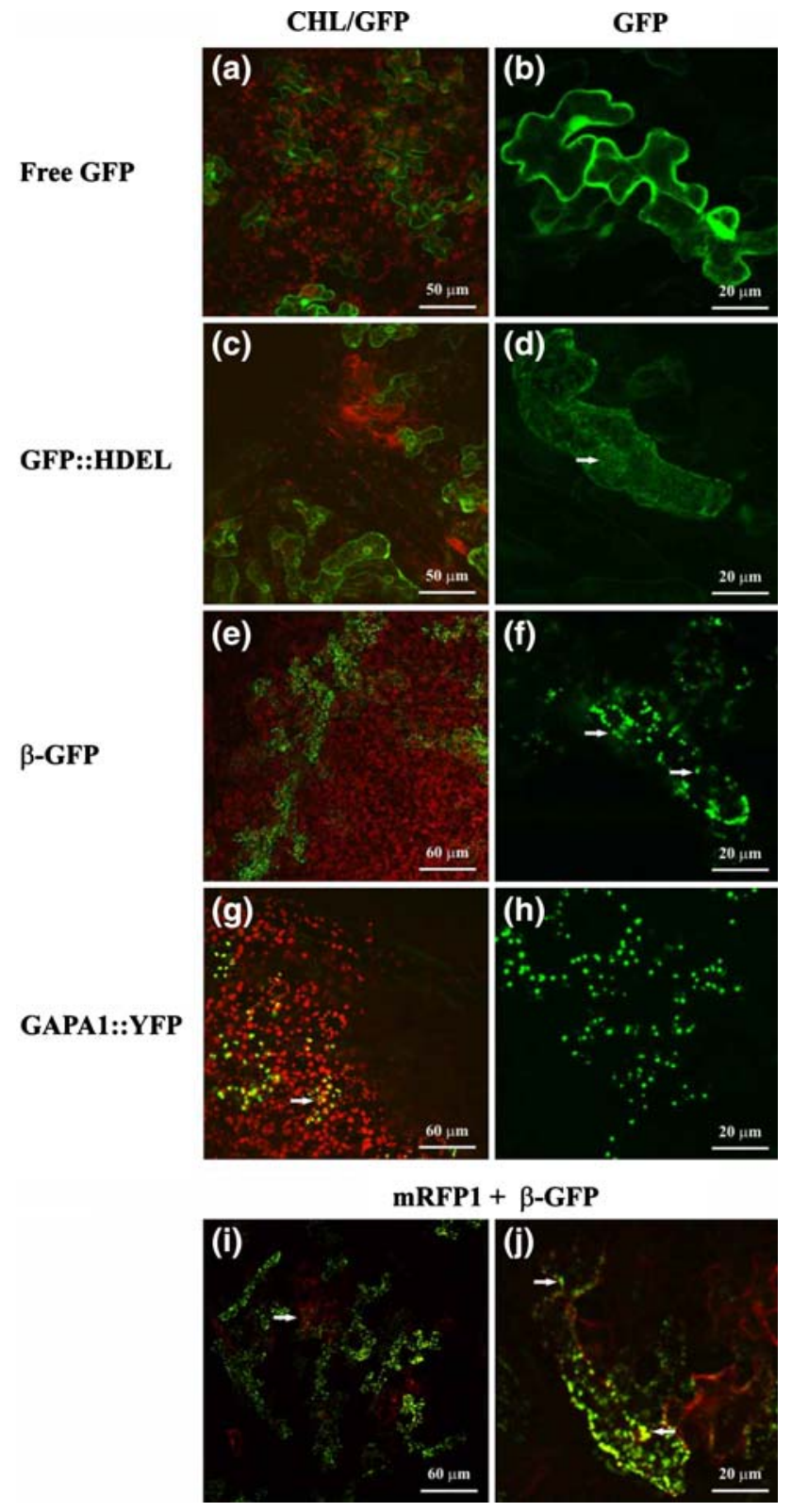

Fig. 5 Subcellular localization of fluorescent proteins analyzed by confocal laser-scanning microscopy. (a-h) Fluorescence imaging of grapevine leaves infiltrated with A. tumefaciens harbouring GFP targeted to different cell compartments: cytosol (a,b), endoplasmic reticulum $(\mathbf{c}, \mathbf{d})$, mitochondria $(\mathbf{e}, \mathbf{f})$, chloroplasts $(\mathbf{g}, \mathbf{h})$. Left column shows images obtained by the overlay of GFP and chlorophyll emission wavelengths; right column shows the single GFP emission signal of the same images. (i-l) Co-transformation experiment. Fluorescence imaging of grapevine leaves infiltrated with $A$. tumefaciens harbouring GFP targeted to mitochondria (green spots) and mRFP localized into the cytosol (red signal). Arrows indicate the regions where the presence of both fluorescence signals is evident

diffuse signal, that corresponds to the free mRFP1 localized into the cytoplasm are clearly recognisable in the same cell. In particular, a cell region where green mitochondria surround a red nucleus, red signal due to free diffusion of the mRFP1 from the cytoplasm to the nucleoplasm can be clearly observed (arrow in Fig. 5i). This result is of particular importance since it demonstrates that co-expression of different proteins occurs in the same cell, which then becomes a useful tool for co-localization experiments or promoter trans-activation studies.

Agroinfiltrated grapevine leaves can be used to produce stable transformed cultured cells

The use of in vitro cultured plants provides the opportunity to maintain leaves sterility after the infiltration experiments. Taking advantage of this, we were able to obtain stable transformed grapevine cell lines starting from agroinfiltrated leaf tissues. In particular, Sugraone leaves were agroinfiltrated with GV3101 harbouring the GFP::HDEL construct (Haseloff et al. 1997). These leaves were used as primary explants (Fig. 6a) to induce a dedifferentiation process by incubating them on a selective "Gamborg" medium supplemented with auxin (see "Material and methods"). The conditions we set up were suitable for inducing transgenic callus production in few weeks (Fig. 6b, c). The use of an Agrobacterium harbouring the GFP::HDEL binary vector allowed us to use the GFP fluorescence as a marker to follow the various steps of callus growth (Fig. 6e, g). In wild type callus (Fig. 6d, h) fluorescence was not detectable. A friable portion of the transgenic callus was transferred to a liquid culture medium to produce a suspension cell culture. After a short period (2-3 weeks) the transferred transgenic cells were able to adapt themselves to the liquid culture conditions and single cell culture lines were obtained (Fig. 6i). The confocal microscope analyses of the transgenic cultured cells confirmed the expression of the GFP::HDEL fusion protein as shown in Fig. 6j, and in fact a clear fluorescent signal was recognisable in all imaged cells. The higher magnification of a transformed cell (Fig. 6k) shows the staining of the ER membranes and the NE which were expected to be marked by the GFP::HDEL fusion protein. The success in selecting stable transformants cells from this type of explant suggests that leaf epidermal cells, usually resistant to dedifferentiation, were not the only transformed cells. It seems that transformed mesophyll or parenchymatic cells constitute an essential source for transgenic callus production. In fact, from subcellular localization analyses performed on agroinfiltrated leaves, we could recognize distinct transformed tissues, and in particular we detected fluorescence mostly in epidermal and mesophyll cells. In addition, we could also see transformed elongated parenchymatic cells associated with leaf vessels (data not shown). 
Fig. 6 Induction of stable transformed callus from agroinfiltrated leaves. (a,e) Primary explant after 3 weeks on selective medium; (b,f) callus formation on leaf primary explant; (c,g) dedifferentiated stable transformed callus; $(\mathbf{d}, \mathbf{h})$ dedifferentiated untransformed control callus. The pictures were acquired using a stereo microscope equipped with white and UV light. (i-k) suspension cell culture pictures acquired with confocal laser-scanning microscopy
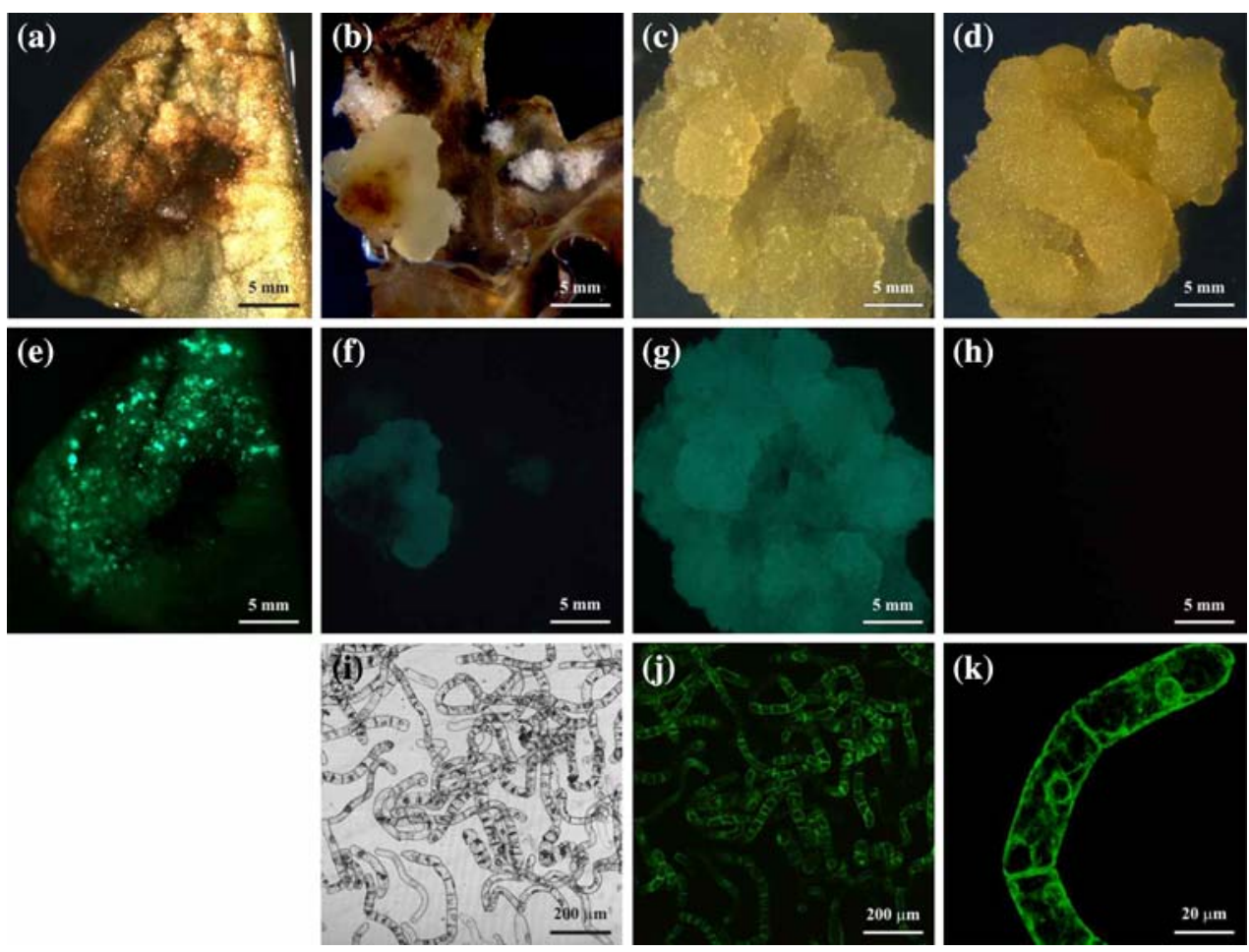

The procedure reported here allows the production of stable transformed cultured cells. However, we did not characterize these cells in terms of maintenance of regeneration capabilities. Nonetheless the advantages of this infiltration procedure in providing transformed cell lines offer simple opportunities for studying gene function and interactions in this economically advantageous system.

\section{Conclusions}

We developed a protocol for efficient and routine transient transformation assays on leaves of Sugraone and Aleatico grapevine cultivars. Transient expression occurred in the cells into the infiltrated area when Sugraone leaves were used. In experiments performed with Aleatico cultivar, transient expression was also observed outside the syringe contact zone.

We observed that the genetic background and the tissue culture conditions were both necessary for a successful agroinfiltration assay in grapevine. We succeeded in performing transient assays in two grapevine cultivars by combining the right plant growth conditions, i.e. tissue culture versus greenhouse, and by selecting appropriate genotypes and compatible bacteria strains.

Acknowledgments We thank Professor Roger Tsien for providing us the pRSET-mRFP1 vector and Prof. Albrecht von Arnim for providing the pAVA554-YFP vector. We thank Dr. Jim Haseloff for providing the pBIN-m-gfp5-ER vector. We thank Prof. Paolo Trost for providing the GAPA1 cDNA clone. This research was supported by the "Progetto di Ateneo" by the University of Padova to M. Z.

\section{References}

Batoko H, Zheng HQ, Hawes C, Moore I (2000) A Rab1 GTPase is required for transport between the endoplasmic reticulum and Golgi apparatus and for normal Golgi movement in plants. Plant Cell 12:2201-2217

Brandizzi F, Fricker M, Hawes C (2002) A greener world: the revolution in plant bioimaging. Nat Rev Mol Cell Biol 3:520 530

Campbell RE, Tour O, Palmer AE, Steinbach PA, Baird GS, Zacharias DA, Tsien RY (2002) A monomeric red fluorescent protein. Proc Natl Acad Sci USA 99:7877-7882

Carimi F, Barizza E, Gardiman G, Lo Schiavo F (2005) Somatic embryogenesis from stigmas and styles of grapevine. In Vitro Cell Dev Biol 41:249-252

Chee R, Pool RM (1987) Improved inorganic media constituents for in vitro shoot moltiplication of Vitis. Sci Hortic 32:85-95

Citovsky V, Kozlovsky SV, Lacroix B, Zaltsman A, Dafny-Yelin M, Vyas S, Tovkach A, Tzfira T (2007) Biological systems of the host cell involved in Agrobacterium infection. Cell Microbiol 9: 9-20

daSilva LL, Snapp EL, Denecke J, Lippincott-Schwartz J, Hawes C, Brandizzi F (2004) Endoplasmic reticulum export sites and Golgi bodies behave as single mobile secretory units in plant cells. Plant Cell 16:1753-1771

Duby G, Oufattole M, Boutry M (2001) Hydrophobic residues within the predicted N-terminal amphiphilic alpha-helix of a plant mitochondrial targeting presequence play a major role in in vivo import. Plant J 27:539-549

Gamborg OL, Miller RA, Ojima K (1968) Nutrient requirements of suspension cultures of soybean root cells. Exp Cell Res 50:151158 
Hamilton RH, Fall MZ (1971) The loss of tumor-initiating ability in Agrobacterium tumefaciens by incubation at high temperature. Experientia 27:229-230

Haseloff J, Siemering KR, Prasher DC, Hodge S (1997) Removal of a cryptic intron and subcellular localization of green fluorescent protein are required to mark transgenic Arabidopsis plants brightly. Proc Natl Acad Sci USA 94:2122-2127

Hellens RP, Edwards EA, Leyland NR, Bean S, Mullineaux PM (2000a) pGreen: a versatile and flexible binary Ti vector for Agrobacterium-mediated plant transformation. Plant Mol Biol 42:819-832

Hellens RP, Mullineaux PM, Klee H (2000b) A guide to Agrobacterium binary Ti vectors. Trends Plant Sci 5:446-451

Hoekema A, Hirsch PR, Hooykaas PJJ, Schilperoort RA (1983) A binary plant vector strategy based on separation of vir- and Tregion of the Agrobacterium tumefaciens Ti-plasmid. Nature 303:179-180

Jones HD, Doherty A, Wu H (2005) Review of methodologies and a protocol for the Agrobacterium-mediated transformation of wheat. Plant Methods 5:1-9

Kapila J, De Rycke R, Van Montagu M, Angenon G (1997) An Agrobacterium-mediated transient gene expression system for intact leaves. Plant Sci 122:101-108

Latz A, Ivashikina N, Fischer S, Ache P, Sano T, Becker D, Deeken $\mathrm{R}$, Hedrich R (2007) In planta AKT2 subunits constitute a $\mathrm{pH}-$ and $\mathrm{Ca}^{2+}$-sensitive inward rectifying $\mathrm{K}+$ channel. Planta 225:1179-1191

Main GD, Reynolds S, Gartland JS (1995) Electroporation protocols in Agrobacterium. In: Gartland KMA, Davey MR (eds) Methods in molecular biology, vol 44: Agrobacterium protocols. Humana Press, Totowa, pp 405-412

Marri L, Trost P, Pupillo P, Sparla F (2005) Reconstitution and properties of the recombinant glyceraldehyde-3-phosphate dehydrogenase/CP12/phosphoribulokinase supramolecular complex of Arabidopsis. Plant Physiol 139:1433-1443
Melotto M, Underwood W, Koczan J, Nomura K, He SY (2006) Plant stomata function in innate immunity against bacterial invasion. Cell 126:969-980

Mezzetti B, Pandolfini T, Navacchi O, Landi L (2002) Genetic transformation of Vitis vinifera via organogenesis. BMC Biotechnol 27:2-18

Murashige T, Skoog F (1962) A revised medium for rapid growth and bioassays with tobacco tissue cultures. Physiol Plant 15:473-497

Schöb H, Kunz C, Meins F Jr (1997) Silencing of transgenes introduced into leaves by agroinfiltration: a simple, rapid method for investigating sequence requirements for gene silencing. Mol Gen Genet 256:581-585

Taiz L, Zeiger E (2006) Plant physiology, 4th edn. Sinauer Associates, Sunderland

The French-Italian Public Consortium for Grapevine Genome Characterization (2007) The grapevine genome sequence suggests ancestral hexaploidization in major angiosperm phyla. Nature 449:463-467

von Arnim AG, Deng XW, Stacey MG (1998) Cloning vectors for the expression of green fluorescent protein fusion proteins in transgenic plants. Gene 221:35-43

Wroblewski T, Tomczak A, Michelmore R (2005) Optimization of Agrobacterium-mediated transient assays of gene expression in lettuce, tomato and Arabidopsis. Plant Biotechnol J 3:259-73

Yang Y, Li R, Qi M (2000) In vivo analysis of plant promoters and transcription factors by agroinfiltration of tobacco leaves. Plant $\mathbf{J}$ 22:543-551

Zhao R, Dielen V, Kinet JM, Boutry M (2000) Cosuppression of a plasma membrane $\mathrm{H}^{+}$-ATPase isoform impairs sucrose translocation, stomatal opening, plant growth, and male fertility. Plant Cell 12:535-546

Zipfel C, Kunze G, Chinchilla D, Caniard A, Jones JD, Boller T, Felix G (2006) Perception of the bacterial PAMP EF-Tu by the receptor EFR restricts Agrobacterium-mediated transformation. Cell 125:749-760 